\title{
An Automated Approach to Modeling Jupiter's Synchrotron Radiation from Radio Telescope Observations
}

\author{
Peyton Robertson ${ }^{1}$, Connor Espenshade ${ }^{1}$, Jay SaRva ${ }^{1}$, OWen \\ DUGAN ${ }^{1}$, AND KALÉE TOCK \\ ${ }^{1}$ Stanford Online High School, CA \\ "Corresponding author: peytonwr@stanford.edu
}

In this study, we used $2.295 \mathrm{GHz}$ radio telescope data to study synchrotron radiation from Jupiter's magnetosphere. We processed scans of Jupiter and calibrators taken by the Goldstone Apple Valley Radio Telescope on various dates, developing and automating algorithms for outlier removal, baseline subtraction, and Gaussian fitting in order to determine the peak intensity of each scan. Comparing the peak intensities of Jupiter to those of the calibrators and the known fluxes of the calibrators, we computed the flux of Jupiter on each scan. Plotting Jupiter's flux against the longitude facing Earth at the time of each scan revealed a periodic relationship between the variables and thus a model for expected synchrotron flux from Jupiter observed at a given longitude. This estimate can corroborate other flux measurements of Jupiter at similar frequencies, such as those taken by the microwave radiometer on the Juno probe.

(ㅁ)(1) $\odot \bigodot 2020$ Astronomy Theory, Observations and Methods Journal

Keywords: Physical data and processes: magnetic fields - Astronomical databases: catalogs

https://doi.org/10.32374/atom.2020.1.3

\section{INTRODUCTION}

On August 5, 2011, NASA launched Juno, a satellite headed for Jupiter to measure key data and answer questions about the the gas giant. The spacecraft's ability to get within close proximity of Jupiter provided the opportunity to acquire unprecedented data, elucidating its atmospheric composition (including the amount of water in its atmosphere), its magnetic and gravitational fields, and the history of its formation. But many of these measurements must take into account the synchrotron radiation due to Jupiter's strong magnetic field and radiation belt (Kollmann, 2017).

In this paper, we investigate how Jupiter's synchrotron radiation varies with the geometry of an observer. Using Earth-based telescopes, we can study the intensity variation as a function of Jupiter's Central Meridian Longitude (CML). This model can then enable more accurate measurements of other radiation (such as those taken by Juno) and the molecules that attenuate it. For example, the microwave and radio intensity of Jupiter is largely attenuated by the 
presence of water and, to a smaller extent, ammonia. Understanding the degree to which the core's thermal radiation is attenuated by these molecules may provide a means of more accurately estimating their concentrations beneath the cloud tops, which could then give clues about the composition and formation of Jupiter's core. Since synchrotron flux is intermingled with the core's thermal radiation, our separate measurement of it provides a means of disentangling its effect.

Understanding how much these molecules attenuate the thermal radiation from the core may provide a greater understanding of how much water and ammonia exists under the cloud tops, which could then give clues about the composition and formation of Jupiter's core.

For Earth-based observations of Jupiter's synchrotron radiation, we used data from the Goldstone Apple Valley Radio Telescope (GAVRT) (Figure 1). The dataset, initially received from Dr. Thangasamy Velusamy, and later queried directly from databases at the Lewis Science Center, contains data from telescope scans of Jupiter, as well as scans of calibrators taken on the same days. These data allow one to compare the telescope offset (varying linearly with time as the telescope sweeps past the target) with the temperature of the antenna (indicating intensity of the signal) during each scan.

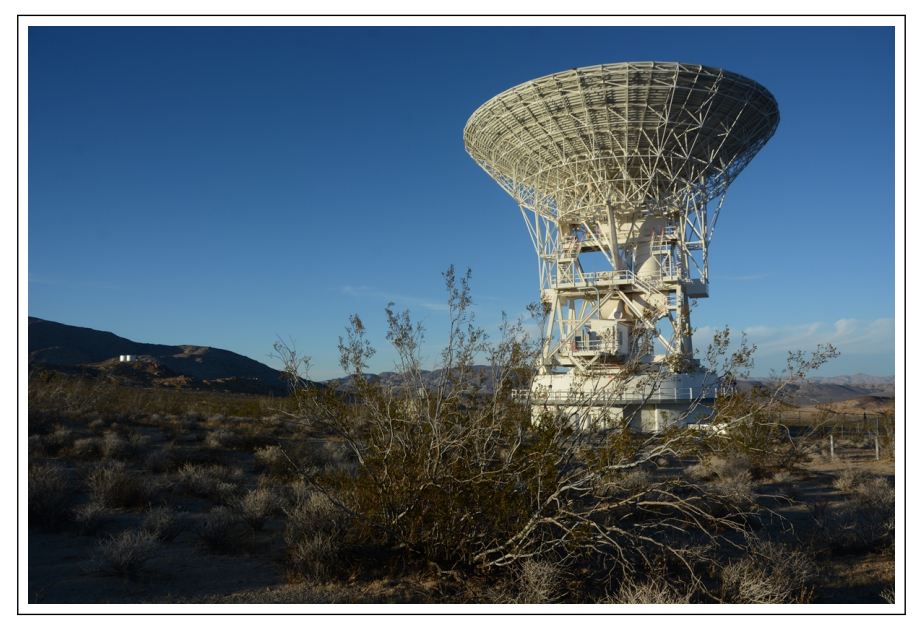

Fig. 1. The Goldstone Apple Valley Radio Telescope (GAVRT) (Goldstone Apple Valley Radio Telescope (GAVRT), n.d.).

\section{SYNCHROTRON RADIATION}

Synchrotron radiation is the result of electrically charged particles following a curved path at relativis- tic velocities (Nave, n.d.). Synchrotron radiation is highly beamed along the forward direction of particle movement.

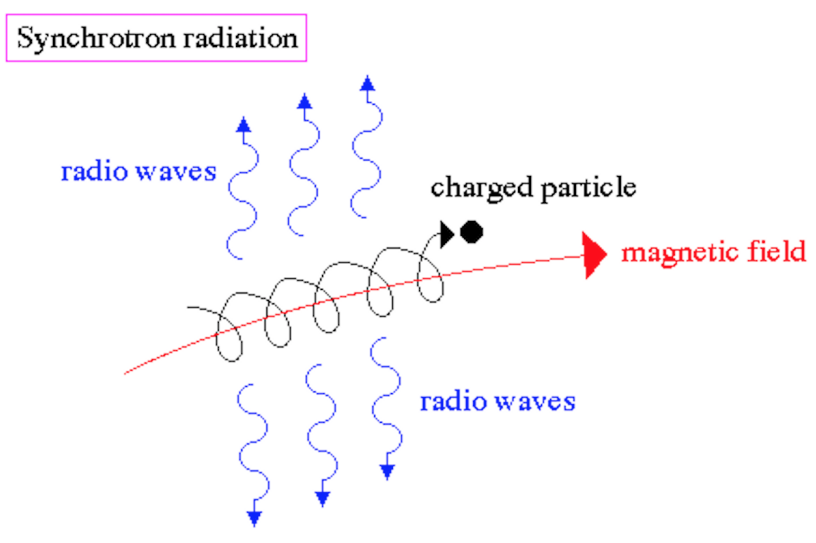

Fig. 2. Diagram showing synchotron emission emitted by electron in the direction of its motion while spiraling around Jupiter's magnetic field lines.

Jupiter has a strong magnetosphere, or magnetic field, which is thought to be produced by metallic hydrogen in its core (Greicius, 2018). Jupiter's high pressure effectively condenses hydrogen gas into a molten flow of elementary particles. The electrons in this flow are accelerated to relativistic speeds as they spiral around Jupiter's magnetic field lines due to Jupiter's magnetosphere (Figure 2). Most spiraling electrons lie in Jupiter's central radiation belt, which is titled relative to the ecliptic. While spiraling around magnetic field lines in the radiation belt, electrons emit synchrotron radiation in the plane of the belt. The orientation of this plane relative to Earth determines if the radiation is observed by Earth-based telescopes, as demonstrated in Figure 3.

As Jupiter rotates (with a period of approximately 9 hours, 56 minutes), an observer from Earth faces not only different longitudes, but also different parts of its radiation belt. When looking directly at the central radiation belt, synchrotron radiation is expected to increase; when looking above or below it, synchrotron radiation is expected to decrease. Due to this wobble effect, we expect to see two minima (a sighting above and below the central radiation belt) and two maxima (two sightings at the radiation belt) each period. The "beaming curve" is a measure of flux as a function of $\mathrm{CML}$; that is, how much radiation is received during scans of Jupiter as the planet rotates. 


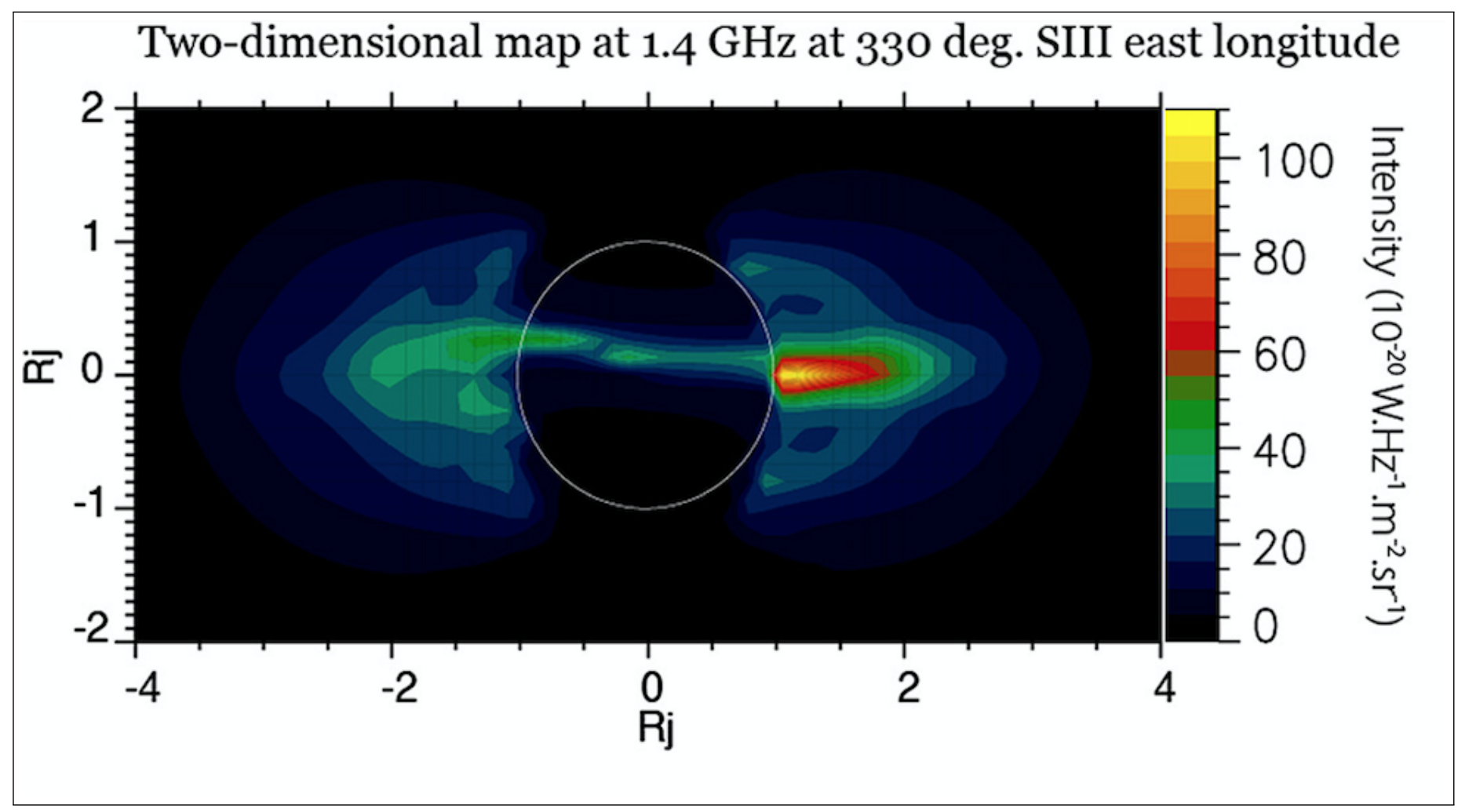

Fig. 3. Map of Jupiter's synchrotron emissions at $1.4 \mathrm{GHz}$ (Santos-Costa et al., 2017). Note that in a single scan of Jupiter, only one peak value is observed due to the geometrical perspective of Earth relative to Jupiter's radiation belt at that point in Jupiter's rotation.

\section{GAVRT SCAN PEAK INTENSITY COMPUTA- TION}

We used scans taken by the GAVRT telescope over January 8, 2018, and January 29-30, 2018.

First, outliers were removed from the scans. For measurements outside the peak (defined by those outside 0.3 degrees of offset from the center offset of 0 ), a measurement was considered an outlier if it fell considerably above or below the adjacent measurements. To implement this mathematically, we multiplied the differences in scan intensity between the measurement and the adjacent measurements if this product lied above some threshold, the measurement was an outlier. Outliers were replaced by the average of the adjacement elements. A threshold of $T=10^{-3}$ seemed to remove a reasonable number of outliers while minimizing the introduction of stray data points.

To remove the baseline, the noise-reduced curve that lay outside of 0.3 degrees of offset from the target was fit to a quadratic, as most scans have baselines that can be well-approximated by a second-degree polynomial. Then this baseline fit was subtracted from the data, which placed almost every measurement that was not a part of the peak near zero.

We fit what was left over after baseline subtraction from each scan to a Gaussian curve, as shown in column 3 of Figure 4.

Let $\mathrm{x}$ represent the offset and $\mathrm{y}$ the noise-reduced, baseline-subtracted flux data. Then the Gaussian distribution is given by three parameters: $a, \mu$, and $\sigma$, where $a$ represents the maximum intensity, $\mu$ represents the offset of the central maximum, and $\sigma$ represents the standard deviation.

$$
y(x)=a e^{\frac{-(x-\mu)^{2}}{2 \sigma^{2}}}
$$

Attempting to fit a Gaussian to each scan led to some inadequate fits that failed to represent the true maximum intensity. For example, at times the peak of the scan occurred far to the right of central offset. Upon performing a live scan controlling the robotic GAVRT telescope, we discovered that such a false peak may occur when the telescope re-centers after scanning past Jupiter. In order to ensure that the peak is located at the central offset, we introduced bounds on the $\mu$ parameter of the Gaussian fit: $-0.3<\mu<$ 

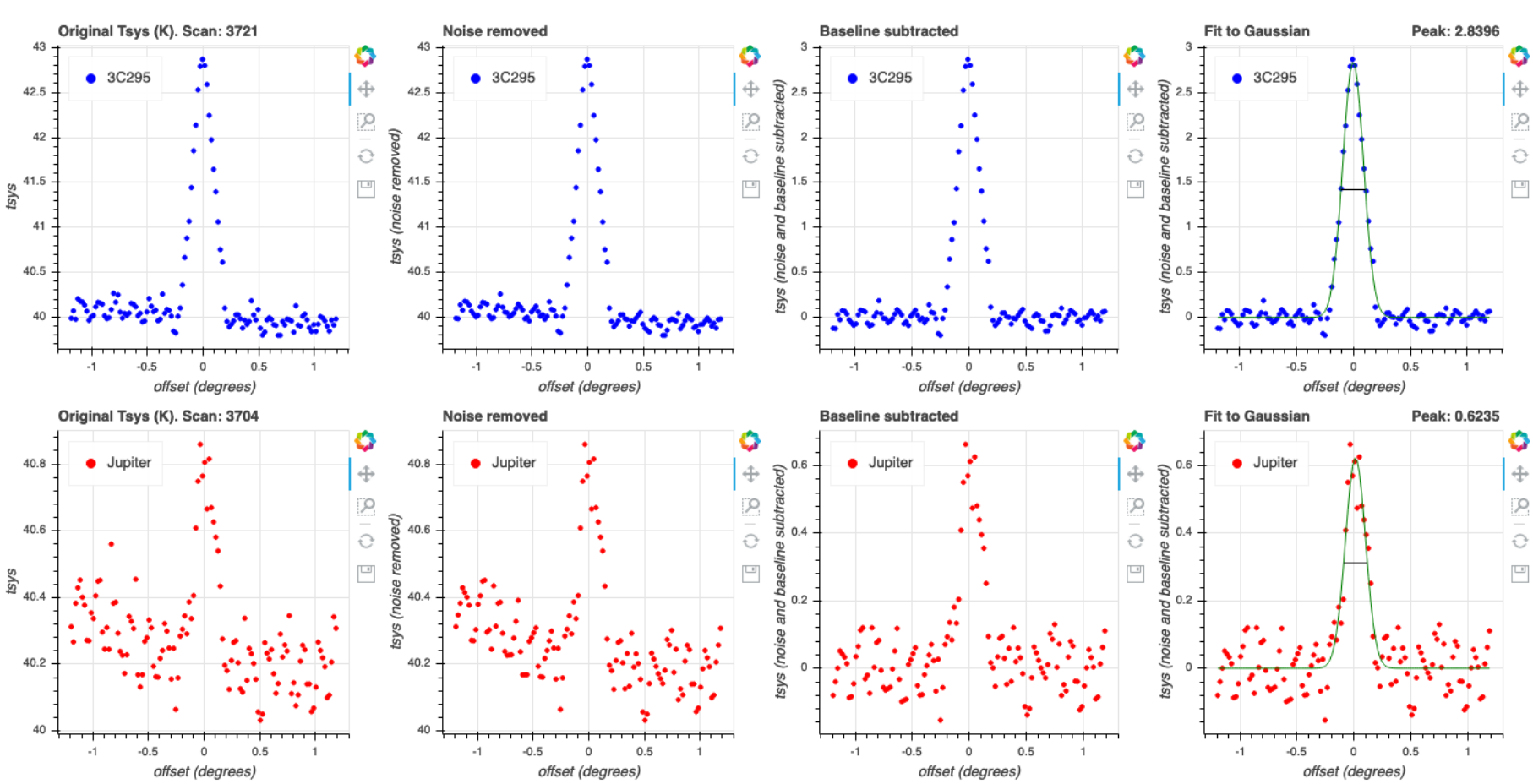

Fig. 4. Some example plots of signal data at each stage of processing (from left to right: original, noise removed, baseline subtracted, Gaussian fit). The top row shows a calibrator scan, while the bottom row shows a Jupiter scan.

0.3 , where $\mu$ represents the offset. Such bounds were enforced through the use of the trust region reflective optimization algorithm.

Similarly, it is important that the half-power width remain reasonably constant between scans. Solving for $\mathrm{x}$ in (1) to find the offset at which the half-intensity is reached, we find

$$
x=\mu \pm \sqrt{-2 \sigma^{2} \ln \frac{y}{a}}
$$

The half-intensity of the curve is $y(\mu) / 2=a / 2$.

The half-power width is the distance between the possible $\mathrm{x}$-values (representing offsets) at this halfintensity, namely

$$
H_{p}=2 \sqrt{2 \ln 2}|\sigma|
$$

Thus the half power varies linearly with $\sigma$, and we may compute it accordingly. Correspondence with Dr. Velusamy suggested that, from the scan frequency of $2.295 \mathrm{GHz}$ and the telescope diameter of $34 \mathrm{~m}$, the half-power width should be fixed to within $20 \%$ of 0.225 . By (2), we have that $\sigma$ must lie within $20 \%$ of 0.0955-these bounds were also incorporated into the curve fitting algorithm.

\section{COMPUTATION OF FLUX}

Before and after scanning Jupiter, telescopes take scans of a calibrator in order to account for telescope area and other environmental factors such as daily weather conditions. The fundamental principle that allows for such a correction is that, at a given time, the peak intensity of a scan of an emitter (in Kelvin) is proportional to the flux of that emitter (in Janskys). We refer to this ratio the antenna gain.

The relevant constant of proportionality for a given measuring device and set of environmental conditions can be determined by scanning a calibrator for which the flux is known. For the January 8 scans, quasar 3C295 was used as a calibrator; on January 29-30, radio galaxy 3C353 was used. The data from quasar 3C295 was later discarded, as this calibrator's suboptimal elevation on the date of the scans seems to have compromised the corresponding measurements, as discussed below.

Let I denote the peak intensity (in Kelvin) and F denote the flux (in Janskys) for a given source. The subscripts 'c' and 'j' denote the calibrator and Jupiter. Then it follows from the antenna gain relation that

$$
\frac{F_{j}}{I_{j}}=\frac{F_{c}}{I_{c}}
$$


Thus we may compute the flux of Jupiter as follows:

$$
F_{j}=I_{j} \frac{F_{c}}{I_{c}}
$$

Using the algorithm in the previous section, we compute the peak intensity for Jupiter $\left(I_{j}\right)$ at each scan, and the mean peak intensity of all calibrator scans on that day $\left(I_{c}\right)$. The mean calibrator intensity is used because there is only one telescope, so the calibrator scans cannot be conducted at precisely the same time as the Jupiter scans. This averaging is acceptable since both calibrators are considered 'stable' sources (Perley \& Butler, 2013).

It remains to find $F_{c}$. From (Perley \& Butler, 2013), we have that the flux density $S$ of a quasar can be approximated as a polynomial expression of the form

$$
\log (S)=\sum_{n=0}^{N} a_{n} \log \left(v_{G}\right)^{n}
$$

where $v G$ is the frequency in GHz. Our scans are taken at a frequency of $2.295 \mathrm{GHz}$. From the coefficients given in the paper, we compute the flux of calibrator 3C295 to be $14.34 \mathrm{Jy}$, and the flux of calibrator 3C353 to be $39.62 \mathrm{Jy}$. From these values and the antenna gain relation, we may compute $F_{j}$ at each scan according to (3).

Across multiple scans, the distance from Earth to Jupiter may vary, affecting the amount of flux received by our telescope by the inverse square of the distance. Although the variation of distances in our dataset is small, it is important that distance be taken into account for future studies with more scans. We normalize all fluxes to the minimum distance of Jupiter from Earth, 4.04 AU. The normalized flux $F_{J}$ can be computed from the distance d from Jupiter to Earth, and the previously computed flux $F_{j}$ by

$$
F_{J}=F_{j}\left(\frac{d}{4.04}\right)^{2}
$$

\section{RETRIEVING EPHEMERIDES DATA}

In order to understand how the flux varies with other features of Jupiter and its orbit, we accessed the HORIZONS Ephemerides data set. We used the astroquery package to retrieve the data with the target body code of Jupiter (599) and the observer location code for the telescope at the Goldstone Deep Space Communications Complex (257). This allowed us to query important data from Jupiter, such as the CML at the time of the scan, and collect them into a table such as Table 1 .

The Central Meridian Longitude in Table 1 "is based on the 'System III' prime meridian rotation angle of the magnetic field" (Giorgini et al., 1996). As the CML changes, the orientation of the magnetic poles relative to an observer viewing the longitude changes as well, thus affecting the magnitude of the synchrotron radiation emitted.

Table 1 shows data from eight example scans, composed by combining the peak intensities as computed from the GAVRT data with CML and Earth-Jupiter distance data queried from the HORIZONS Ephemerides database.

\section{MODELING SYNCHROTRON FLUX AS A FUNC- TION OF CML}

Figure 5 shows Jupiter's flux (both scaled and unscaled for the Jupiter-Earth distance) plotted against the Central Meridian Longitude at each scan, thus producing a rough model of Jupiter's synchrotron radiation as a function of longitude.

The fluxes measured on January 8 using calibrator 3C295 do not produce a smooth curve, suggesting that it is not an appropriate calibrator, likely due to its high elevation relative to Jupiter. The elevations are compared in Table (2).

The two largest outliers are removed in both plots. We may justify their removal through their poor Gaussian fits and thus inaccurate peak intensity, as shown in Figure 6.

To generate a continuous beaming curve, we fit a polynomial model to the 3C353 data. While there was not sufficient data to establish a true periodic fit from a Fourier basis (sum of trigonometric functions), we required the polynomial fit to be semi-periodic - that is, have equal first derivatives at its endpoints: $f(0)=$ $f(1)$ and $f^{\prime}(0)=f^{\prime}(1)$. The coefficients with these constraints imposed were determined by LevenbergMarquardt least squares optimization to minimize residuals. Let $\mathrm{c}$ represent CML measured in degrees. Then the flux $f(c)$ as a best-fitting polynomial is given by (4), and plotted in Figure 7 (left). 


\begin{tabular}{cccccc}
\hline Scan & CML $^{\circ}{ }^{\circ}$ & Jupiter-GAVRT (AU) & peak intensity (K) & Raw Flux (Jy) & Normalized Flux (Jy) \\
\hline 4775 & 204.0 & 5.5174 & 0.68 & 3.40 & 6.34 \\
4776 & 205.5 & 5.5174 & 0.57 & 3.42 & 6.37 \\
4777 & 208.4 & 5.5174 & 0.63 & 3.49 & 6.52 \\
4778 & 209.8 & 5.5173 & 0.60 & 3.42 & 6.39 \\
4779 & 212.1 & 5.5173 & 0.71 & 3.52 & 6.56 \\
4780 & 213.6 & 5.5173 & 0.67 & 3.40 & 6.35 \\
4781 & 215.5 & 5.5172 & 0.69 & 3.51 & 6.55 \\
4782 & 216.9 & 5.5172 & 0.70 & 3.47 & 6.48
\end{tabular}

Table 1. Example scans with fluxes computed from integrated HORIZONS and GAVRT data.

\begin{tabular}{ccc}
\hline Source & Mean Elevation & Std. Dev. Elevation \\
\hline 3C295 & 61.0 & 9.5 \\
3 C353 & 43.1 & 10.1 \\
Jupiter & 33.6 & 4.3
\end{tabular}

Table 2. Elevations of Jupiter and Calibrator Quasars
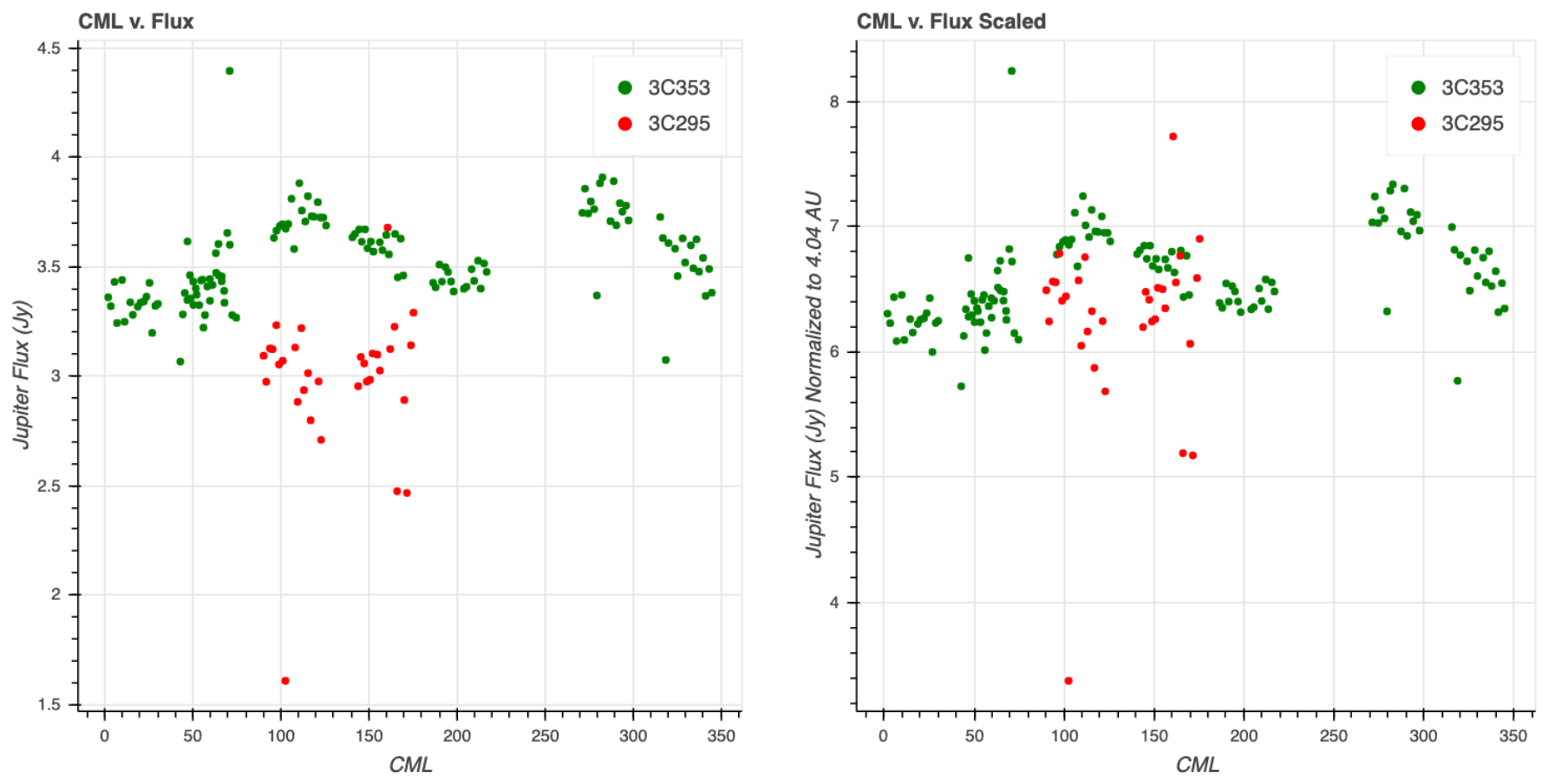

Fig. 5. Plots of CML v. Flux of Jupiter's synchrotron radiation measured with different calibrators. (Calibrator 3C295 used on January 8, 2018; Calibrator 3C353 used on January 29-30, 2018.) The right plot is scaled for inverse-square of Jupiter-Earth distance. 

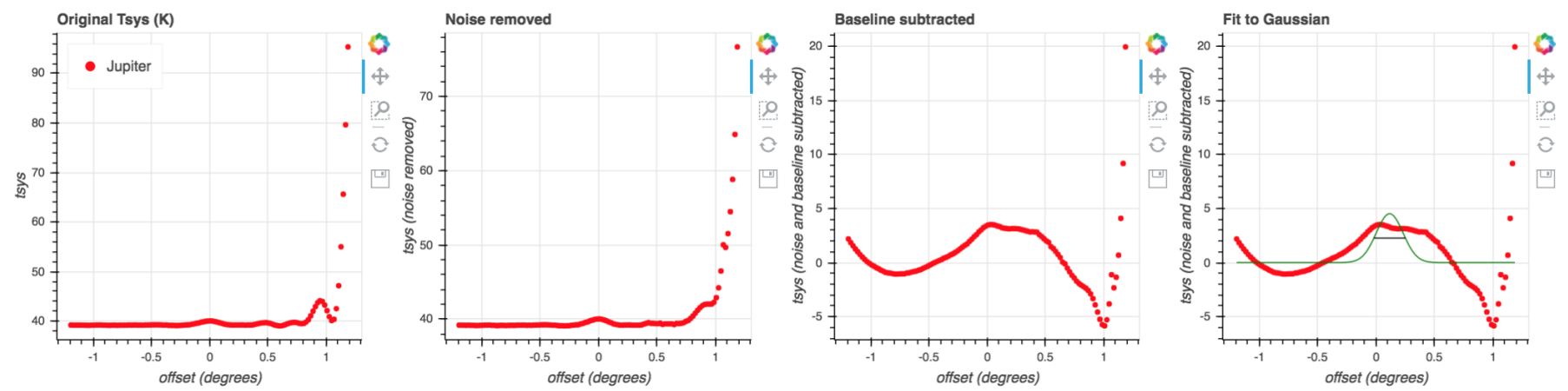

4655.0
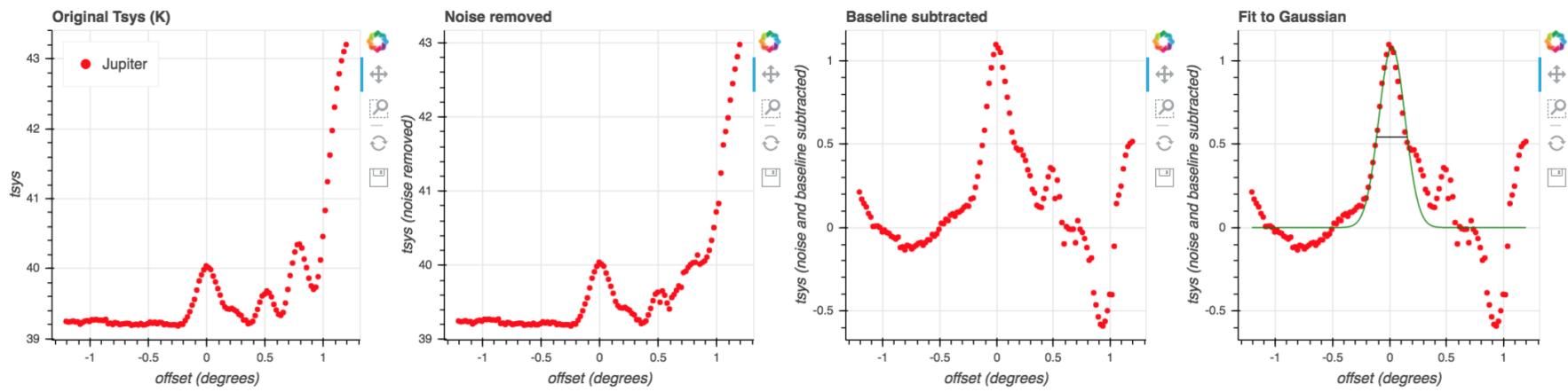

Fig. 6. Scans with poor Gaussian fits and outlying peak intensities, shown at each stage of processing (from left to right: original, noise removed, baseline subtracted, Gaussian fit).

$$
\begin{aligned}
f(c)= & 906.734\left(\frac{c}{360}\right)^{7}-2961.63\left(\frac{c}{360}\right)^{6} \\
& +3638.27\left(\frac{c}{360}\right)^{5}-2056.70\left(\frac{c}{360}\right)^{4} \\
& +514.6\left(\frac{c}{360}\right)^{3}-42.66\left(\frac{c}{360}\right)^{2} \\
& +1.472\left(\frac{c}{360}\right)+6.189
\end{aligned}
$$

The polynomial function fit well, with a residual square sum (RSS) of 7.94. However, we also believed a sinusoidal function would have made more logical sense in an astronomical context. A piecewise sinusoidal function was manually found to fit the data, given by (5) and shown in Figure 7, with a worse residual sum of squares of 9.23 The piecewise function was necessary as the plot has different amplitudes for the first and second peaks. Residual plots for each curve are compared in Figure 8.

$$
f(c)= \begin{cases}-0.44 \cos (0.037(c-35))+6.72 & c<130 \\ -0.37 \cos (0.037(c-197))+6.82 & c \geq 130\end{cases}
$$

We believe the trigonometric piece-wise function (5) could be beneficial with more data as it makes more intuitive sense given the periodic nature of the system, but the seventh-degree polynomial fit (4), with a lower RSS and its continuity, should be used for this dataset. We also attempted to find a twofrequency model of the form $a_{0}+a_{1} \cos \left(b_{1}\left(x-\delta_{1}\right)\right)+$ $a_{2} \cos \left(b_{2}\left(x-\delta_{2}\right)\right)$, but were unsuccessful in finding an adequate fit.

The minima occur at $0^{\circ}$ and $208^{\circ}$ for the polynomial and $35^{\circ}$ and $267^{\circ}$ in the trigonometric model. Despite the better fit of the polynomial model, the model constructed by Levin et al. place the first minima at approximately $35^{\circ}$, agreeing with the trigonometric fit (Levin, Bolton, Gulkis, \& Klein, 2001).

\section{FUTURE WORK}

Volcanic activity on Io may interfere with Jupiter's magnetic field, which could account for other unexplained variation in fluxes at the same longitude between scan dates (Observing Jupiter Radio Storms: Past, Present, and Future, n.d.). Radio bursts from Io in decametric wavelengths are known to affect electrons in Io's field (Clarke et al., n.d.). For future research, it may be useful to compare Jupiter's synchrotron flux at a given longitude with the relative location of Io. It would be necessary to remove the beaming curve, the flux density as a function of CML, 
CML v. Flux Scaled

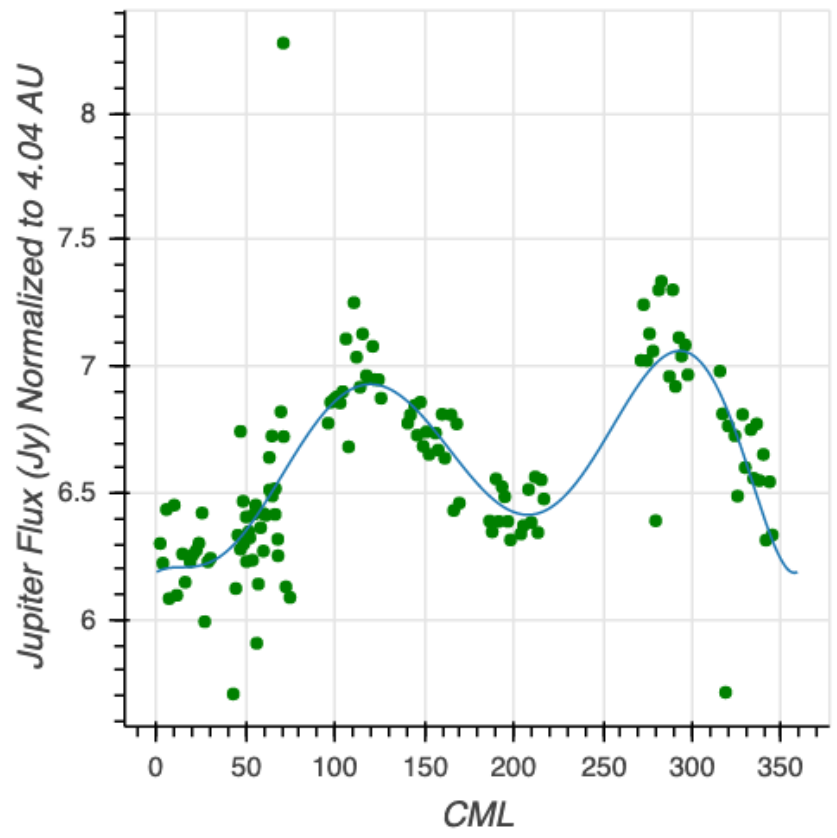

CML v. Flux Scaled

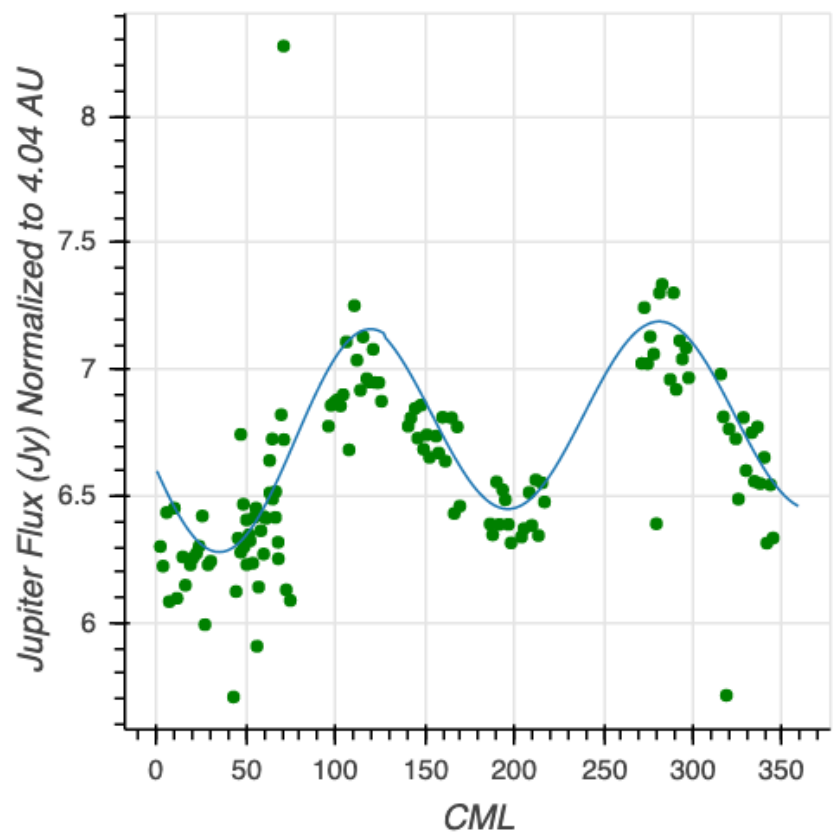

Fig. 7. Beaming Curve Fits: Semi-periodic polynomial fit from equation (4) (left); Sinusoidal piece-wise regression for the Flux vs. CML plot (5) (right).
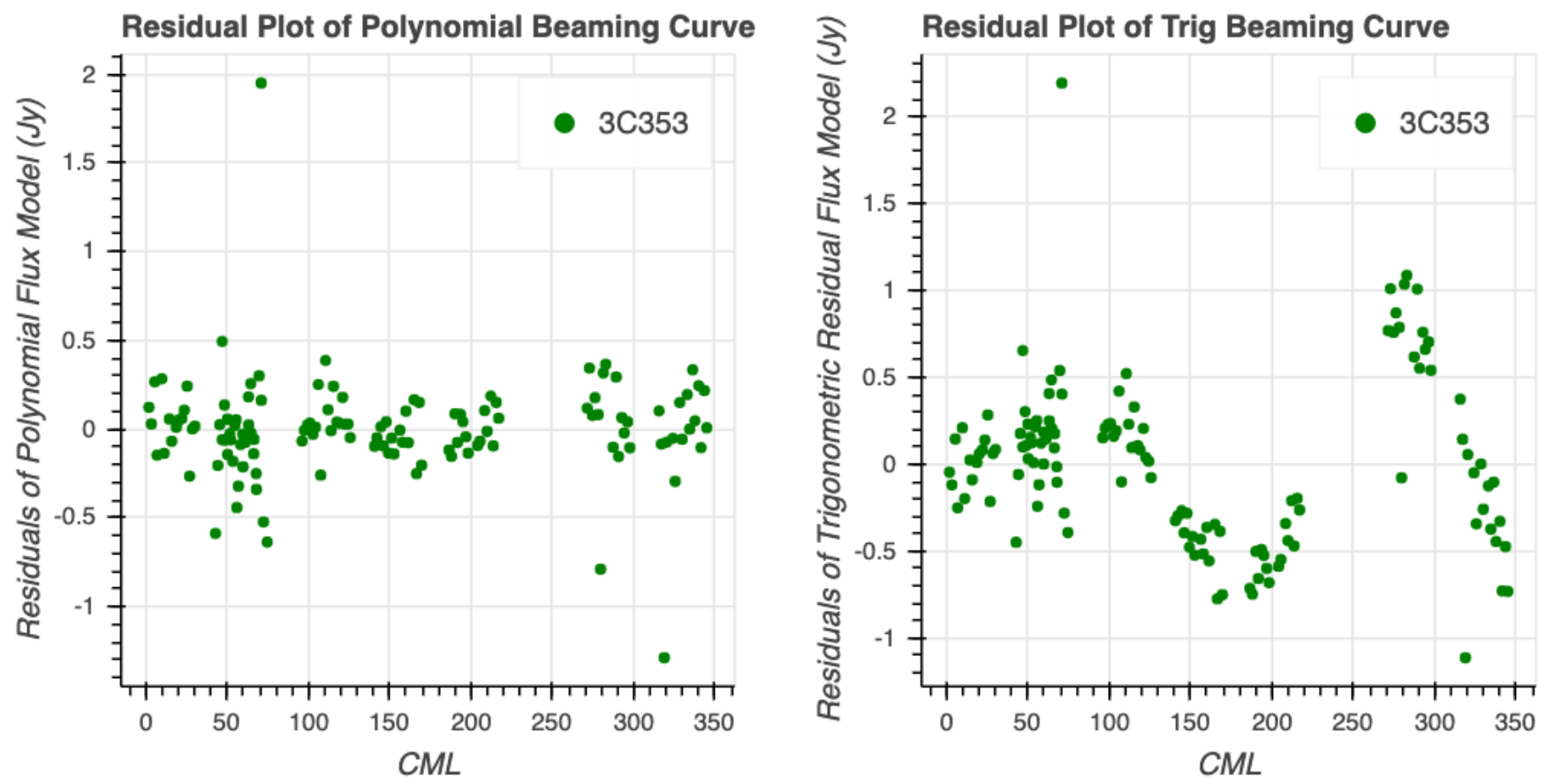

Fig. 8. Residual plots of semi-periodic polynomial model (left) and piecewise trigonometric model (right).

to see this. To demonstrate how such an investigation might proceed, we queried the line of longitude on Jupiter nearest to Io's location at each scan time, and computed the difference between Io's Longitude and the CML of Jupiter that is facing Earth. We plotted this longitude difference against the residuals of the beaming curve, but no general trend was observed. If Io did significantly affect the intensity of synchrotron radiation, we would expect to observe a peak at 0 in Figure 9. However, we had a limited range of dates 
of GAVRT data; further research should of course be conducted with a larger dataset.

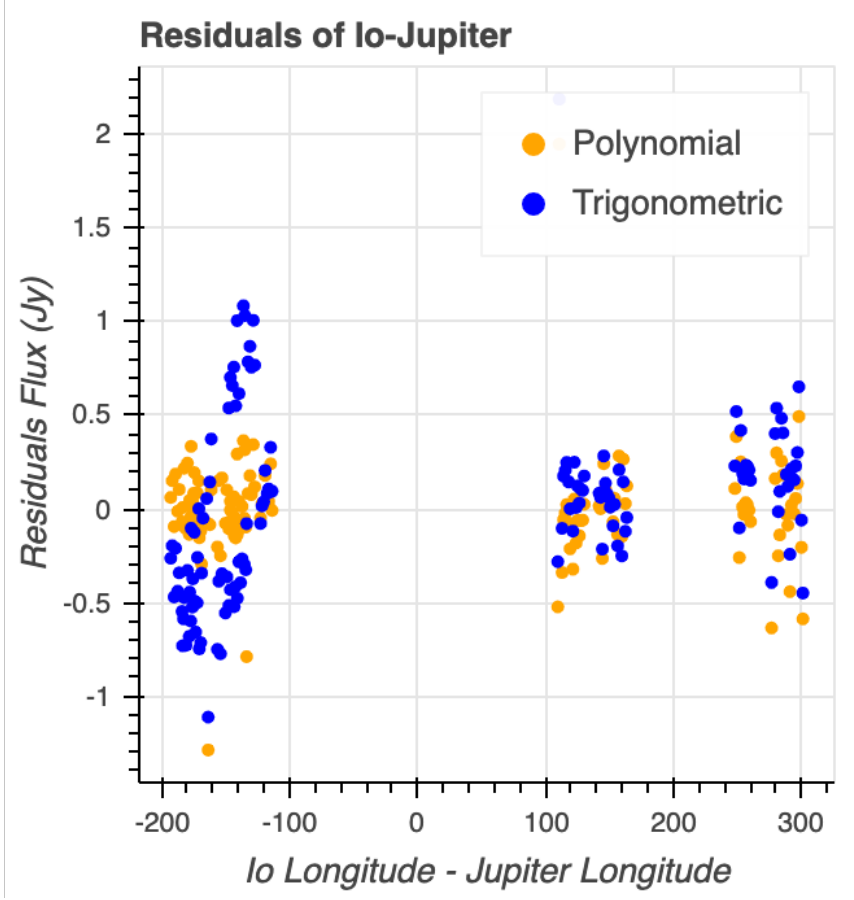

Fig. 9. Longitude Difference between the Io and Jupiter plotted against the residual between the estimated and actual flux from each function.

UV radiation from the Sun may also affect Jupiter's radiation belt and thus synchrotron emission. While solar flares and coronal mass ejections would take years to reach Jupiter and have a noticeable effect, UV radiation only would take approximately 43 minutes (traveling at light speed). Thus the effect of $\mathrm{UV}$ radiation (reaching Jupiter at various intensities as its orbital radius changes) could be investigated by examining a beaming curve such as that of Figure 8 observed over many more periods of Jupiter's rotation.

\section{CONCLUSION}

We developed code to model the flux of synchrotron radiation in Jupiter's magnetosphere as a function of longitude. We implemented procedures for noise removal, baseline subtraction, and Gaussian fitting to find each scan's peak intensity. We then found the antenna gain ratio in order to compute Jupiter's flux from its scan intensity, as well as the scan intensities and known flux of each calibrator quasar. We scaled the flux for distance so fluxes can be compared between other scans when Jupiter is closer or farther from Earth. We found a periodic relationship between Jupiter's longitude and synchrotron flux, and fit a predictive mathematical model to this end. This model may help to inform the amount of $2.295 \mathrm{GHz}$ radiation contributed by various sources to the measurements taken by the Juno probe.

\section{ACKNOWLEDGEMENTS}

Special thanks to Dr. Velusamy Thangasamy for providing the GAVRT dataset and explaining his methods for processing it.

Special thanks to the GAVRT staff for providing training sessions and dedicating time to allow us to use the Goldstone Apple Valley Telescope alongside providing useful information relevant to the project.

Special thanks to astronomer Robert Buchheim as well for his thoughtful feedback and suggestions.

\section{REFERENCES}

Clarke, T., Higgins, C., Skarda, J., Imai, K., Imai, M., Reyes, F., ... Taylor, G. (n.d.). Probing jovian decametric emission with the long wavelength array station 1. Journal of Geophysical Research.

Giorgini, J., Yeomans, D., Chamberlin, A., Chodas, P., Jacobson, R., Keesey, M., ... Wimberly, R. (1996). Jpl's on-line solar system data service. Bulletin of the American Astronomical Society, 28(3).

Goldstone apple valley radio telescope (gavrt). (n.d.). NASA. Retrieved from https://www.nasa.gov/ directorates/heo/scan/communications/ outreach/gavrt

Greicius, T. (2018). Juno overview. Retrieved from https://www.nasa.gov/mission_pages/ juno/overview/index.html (Last accessed 18 January 2019)

Kollmann, e. a., P. (2017). A heavy ion and proton radiation belt inside of jupiter's rings. Geophys, 44, 5259-5268. Retrieved from https://doi . org/10.1002/2017GL073730 doi:

Levin, S. M., Bolton, S. J., Gulkis, S. L., \& Klein, M. J. (2001). Modeling jupiter's synchrotron radiation. Geophysical Research Letters, 28.

Nave, R. (n.d.). Synchrotron radiation. Retrieved from http://hyperphysics.phy-astr.gsu.edu/ hbase/Particles/synchrotron.html (Last accessed 18 January 2019)

Observing jupiter radio storms: Past, present, and future. (n.d.). NASA. 
Perley, R. A., \& Butler, B. J. (2013). An accurate flux density scale from 1 to $50 \mathrm{ghz}$. The Astrophysical Journal Supplement Series, 204.

Santos-Costa, D., Adumitroaie, V., Ingersoll, A., Gulkis, S., Janssen, M. A., Levin, S. M., .. Connerney, J. E. P. (2017). First look at jupiter's synchrotron emission from juno's perspective. Geophysical Research Letters, 44(17), 8676-8684. Retrieved from https: //agupubs . onlinelibrary. wiley .com/doi/abs/10.1002/2017GL072836 doi: 